\title{
Searching for Magnetospheres around Herbig Ae/Be Stars
}

\author{
Mikhail Pogodin $1, *\left(\mathbb{D}\right.$, Natalia Drake ${ }^{2,3}{ }^{\oplus}$, Nina Beskrovnaya ${ }^{1, *} \mathbb{(}$, Sergei Pavlovskiy ${ }^{1}$, Swetlana Hubrig ${ }^{4}$, \\ Markus Schöller ${ }^{5}$, Silva Järvinen ${ }^{4} \mathbb{\oplus}$, Olesya Kozlova ${ }^{6}$ and Ilya Alekseev ${ }^{6}$
}

1 Central Astronomical Observatory at Pulkovo, 196140 Saint-Petersburg, Russia; sergpavlovsky@gmail.com

2 Laboratory of Observational Astrophysics, Saint Petersburg State University, Universitetski pr. 28, 198504 Saint-Petersburg, Russia; natalia.drake.2008@gmail.com

3 Laboratório Nacional de Astrofisica/MCTI, Rua dos Estados Unidos 154, Itajuba 37504-364, Brazil

4 Leibniz-Institut für Astrophysik Potsdam (AIP), An der Sternwarte 16, 14482 Potsdam, Germany; shubrig@aip.de (S.H.); sjarvinen@aip.de (S.J.)

5 European Southern Observatory (ESO), Karl-Schwarzschild-Str. 2, 85748 Garching, Germany; mschoell@eso.org

6 Crimean Astrophysical Observatory, Russian Academy of Sciences, 298409 Nauchny, Russia; oles_kozlova@mail.ru (O.K.); ilya-alekseev@mail.ru (I.A.)

* Correspondence: mikhailpogodin@mail.ru (M.P); beskrovnaya@yahoo.com (N.B.)

Citation: Pogodin, M.; Drake, N.; Beskrovnaya, N.; Pavlovskiy, S.; Hubrig, S.; Schöller, M.; Järvinen, S.; Kozlova, O.; Alekseev, I. Searching for Magnetospheres around Herbig Ae/Be Stars. Universe 2021, 7, 489. https: / / doi.org/10.3390/ universe7120489

Academic Editors: Galina L. Klimchitskaya, Vladimir M. Mostepanenko and Nazar R. Ikhsanov

Received: 1 November 2021 Accepted: 9 December 2021 Published: 12 December 2021

Publisher's Note: MDPI stays neutral with regard to jurisdictional claims in published maps and institutional affiliations.

Copyright: (c) 2021 by the authors. Licensee MDPI, Basel, Switzerland. This article is an open access article distributed under the terms and conditions of the Creative Commons Attribution (CC BY) license (https:// creativecommons.org/licenses/by/ $4.0 /)$.
Abstract: We describe four different approaches for the detection of magnetospheric accretion among Herbig Ae/Be stars with accretion disks. Studies of several unique objects have been carried out. One of the objects is the Herbig Ae star HD 101412 with a comparatively strong magnetic field. The second is the early-type Herbig B6e star HD 259431. The existence of a magnetosphere in these objects was not recognized earlier. In both cases, a periodicity in the variation of some line parameters, originating near the region of the disk/star interaction, has been found. The third object is the young binary system HD 104237, hosting a Herbig Ae star and a T Tauri star. Based on the discovery of periodic variations of equivalent widths of atmospheric lines in the spectrum of the primary, we have concluded that the surface of the star is spotted. Comparing our result with an earlier one, we argue that these spots can be connected with the infall of material from the disk onto the stellar surface through a magnetosphere. The fourth example is the Herbig Ae/Be star HD 37806. Signatures of magnetospheric accretion in this object have been identified using a different method. They were inferred from the short-term variability of the He I $\lambda 5876$ line profile forming in the region of the disk/star interaction.

Keywords: Herbig Ae/Be stars; disk accretion; magnetosphere; individual: HD 10141; HD 259431; HD 104237; HD 37806

\section{Introduction}

Herbig Ae/Be stars (HAeBes) are widely recognized as pre-main sequence (PMS) objects with pronounced emission line features and a far-infrared (FIR) excess indicative of cool dust in their accretion disks [1-3]. These stars are intermediate-mass $\left(2-8 M_{\odot}\right)$ analogues of T Tauri K-M stars. According to current views, the HAeBes have no convective interiors that could support classical dynamo actions as found in the fully convective $\mathrm{T}$ Tauri stars (e.g., [4]). For this reason, strong magnetic fields (such as $\sim 10^{3} \mathrm{G}$ for T Tauri stars) are not expected in HAeBes. On the other hand, over the last years a number of spectropolarimetric studies revealed that about $20 \%$ of the Herbig Ae/Be stars have globally organized magnetic fields of the order of $100 \mathrm{G}$ ([5-11], and some other similar works).

The question of the origin of magnetic fields in intermediate mass stars with radiative envelopes is still under debate. It has been argued that these fields could be fossil relics of fields that are present in the interstellar medium from which the stars were formed (e.g., Moss [12]). However, the fossil field hypothesis also faces problems (see, e.g., the review by Hubrig et al. [13]). 
Notably, the traditional spectropolarimetric method of stellar magnetic field diagnostics has some drawbacks:

- It is effective only for objects with small projected rotation velocity $v \sin i$, because the method is based on the measurement of the fine Zeeman splitting in the presence of a magnetic field, which is difficult to detect if the line broadening due to stellar rotation is significant.

- This method allows one to measure only the mean longitudinal magnetic field $\left\langle B_{z}\right\rangle$, which is the projection of the magnetic field vector on the line of sight averaged over the stellar limb. As a rule, this value is more than three times smaller than the modulus of the surface magnetic field $B$. It is necessary to repeat the spectropolarimetric measurements on different phases of the stellar rotation, and to use modeling to reconstruct the complete configuration of the global magnetic field of the object and to estimate its strength.

This work presents a review of several of our investigations, in which other methods to study magnetic fields in the Herbig Ae/Be stars were proposed. It is based on a search for any signs of magnetospheric accretion from a disk onto a star. These methods can be applied in cases where a direct spectropolarimetric measurement of the magnetic field is inefficient, in particular, if the star is rapidly rotating.

\section{Magnetospheres of Herbig Ae/Be Stars}

We propose an alternative method to study the magnetism of stars with accretion disks such as T Tauri and Herbig Ae/Be stars. This method is related to the search of magnetospheres around such objects, where a specific structure of the circumstellar (CS) environment is observed in the region of the disk/star interaction.

According to the magnetospheric accretion (MA) scenario, if a star possesses a sufficiently strong magnetic field, the transfer of accreted material from the disk to the star is stopped by the magnetic field at a certain distance from the star, the Alfvén radius $R_{\mathrm{A}}$, where the magnetic and kinematic energy densities become equal. The zone around the star at distances $r<R_{\mathrm{A}}$ is called the magnetosphere. Inside the magnetosphere the motion of the accreted gas is completely governed by the magnetic field. A portion of the material is funneled from the disk toward the star along the closed magnetic force lines in the region near the magnetic poles, while other material is flowing outwards along the open force lines at lower magnetic latitudes. An example of magnetic field topology near the magnetosphere of a T Tauri star is presented in Figure 12 from [14].

While models of magnetically driven accretion and outflows successfully reproduce many observational properties of the classical T Tauri stars, the picture is unclear for the HAeBes, since we have poor knowledge of whether their magnetic fields are reasonably strong to maintain the MA scenario of the disk/star interaction. Thus, the detection of a magnetosphere in HAeBes can be an important indicator for significant magnetic fields in these objects.

According to the well-known equation connecting the magnetospheric radius of the star, $R_{\mathrm{A}}$, with other stellar parameters such as the magnetic field strength $B$, the radius $R$, the mass $M$ and the mass accretion rate $\dot{M}$ [15]:

$$
R_{\mathrm{A}}=\left(\mu^{2} / \dot{M}(2 G M)^{1 / 2}\right)^{2 / 7},
$$

where $\mu=B R^{3}$ denotes the star's dipole moment.

Assuming that the observational signs of a magnetosphere can be revealed if $R_{\mathrm{A}} / R>1.5$ and adopting a mean value of the mass accretion rate $\dot{M}$ for HAeBes equal to $5 \times 10^{-7} M_{\odot} / \mathrm{yr}$ [16], and the solar value for the ratio $M / R$, we can estimate a lower limit of $B>500 \mathrm{G}$ for $R=2.5 R_{\odot}$ (Herbig Ae stars) and $B>200 \mathrm{G}$ for $R=6 R_{\odot}$ (Herbig Be stars). These values of $B$ appear quite reasonable for the HAeBes.

Provided the magnetic axis of the star is not coincident with the axis of stellar rotation (i.e., a magnetic oblique rotator), the geometrical configuration inside the magnetosphere 
is not axially symmetric relative to the rotation axis. As a result, a global azimuthal inhomogeneity is formed in the CS environment near the star. In this case, the rotation of the star with its magnetosphere modulates different observational parameters and stimulates their cyclic variability with the period $P=P_{\text {rot }}$ (rotation period) or $P_{\text {rot }} / 2$, depending on whether one or two magnetic poles can be seen during one rotation cycle.

The majority of the magnetosphere diagnostics are based on a search for such cyclic variability. Two types of inhomogeneity responsible for the generation of this variability are: (a) an accretion stream inside the magnetosphere, asymmetric relative to the rotation axis and (b) a hot spot on the stellar surface in the region where the accreted material falls onto the star. The first of them can be discovered by spectroscopy in lines originating near the region of the disk/star interaction. The local hot spots on the stellar surface can be revealed by means of photometry and high resolution spectroscopy of high accuracy using atmospheric lines sensitive to the temperature.

In this work, we present four examples of a successful detection of magnetospheres around Herbig Ae/Be stars.

\section{The Magnetic Herbig Ae Star HD 101412}

This Herbig A2e star has been chosen to test our approach in searching for signatures of magnetospheres as this object is certain to have a magnetosphere: its surface magnetic field was estimated as $B \sim 3 \mathrm{kG}$, which is a rather large value among HAeBes [17]. The magnetic field configuration of HD 101412 has a unique orientation relative to the observer: the angle between the line of sight and the rotation axis $i=80^{\circ} \pm 7^{\circ}$ and the angle between the rotation and magnetic axes has been estimated as $\beta=84^{\circ} \pm 13^{\circ}$. This implies that the magnetic poles are close to the accretion disk plane and the areas around the magnetic poles, where the accreted matter falls onto the star, are seen twice per rotation period, which was estimated as $P_{\text {rot }}$ or $P_{\mathrm{m}}=42.076 \pm 0 \mathrm{~d} 017$. An artist's impression of the MA-scenario in HD 101412 is presented in Figure 1.

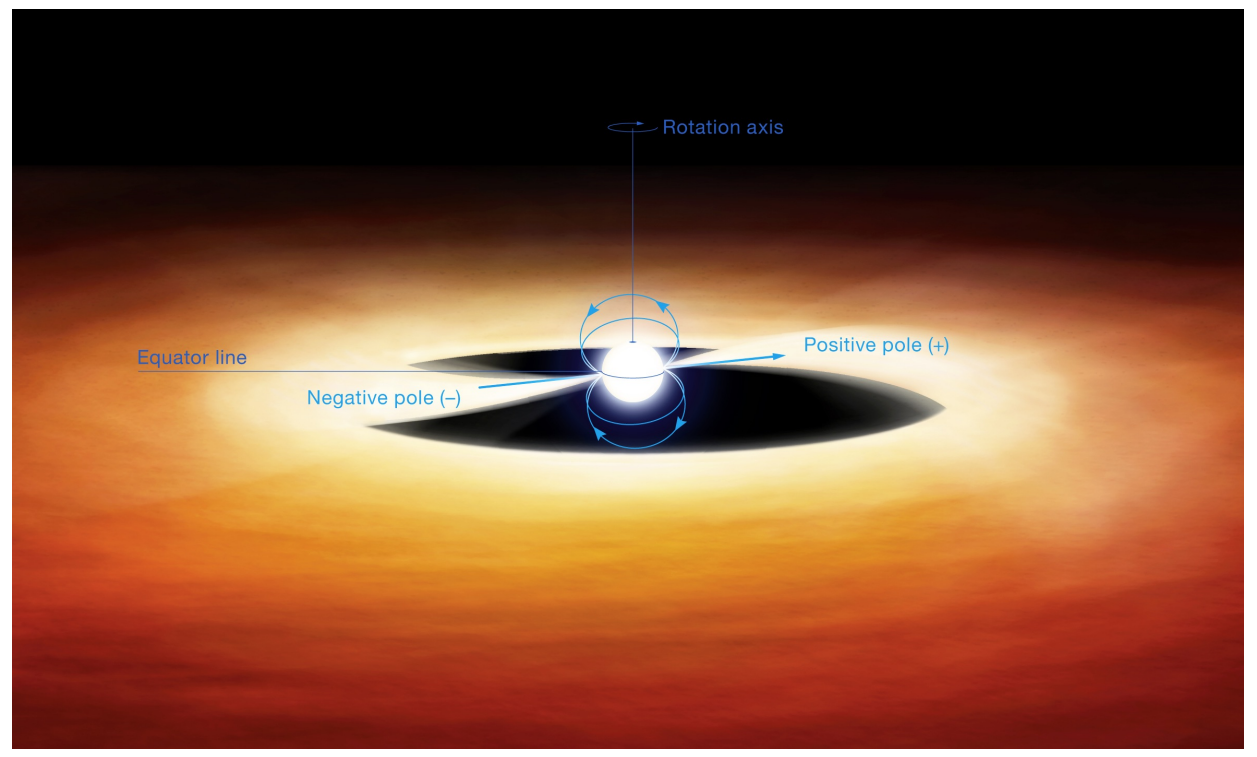

Figure 1. Artist's impression of the MA in HD 101412, looking at the magnetic equator. The exact size and shape of both the disk and the accretion streams are for illustration only, since we lack constraints on these parameters [18]. Credit: Schöller et al., A\&A, 592, A50, 2016, reproduced with permission (C ESO.

We obtained 30 spectra of HD 101412 between 2011 and 2014 with the spectrographs CRIRES and X-shooter, installed at the 8-m telescopes of the Very Large Telescope (VLT) at ESO (Chile). The spectra covered the near infrared (NIR) spectral range, including the lines He I $\lambda 10830$ and $\mathrm{Pa} \gamma$. Both lines are formed in the CS environment close to the accretion 
region, and their parameters are expected to be modulated by the rotation of the star and its magnetosphere. Typical profiles of these two lines are shown in Figure 2.

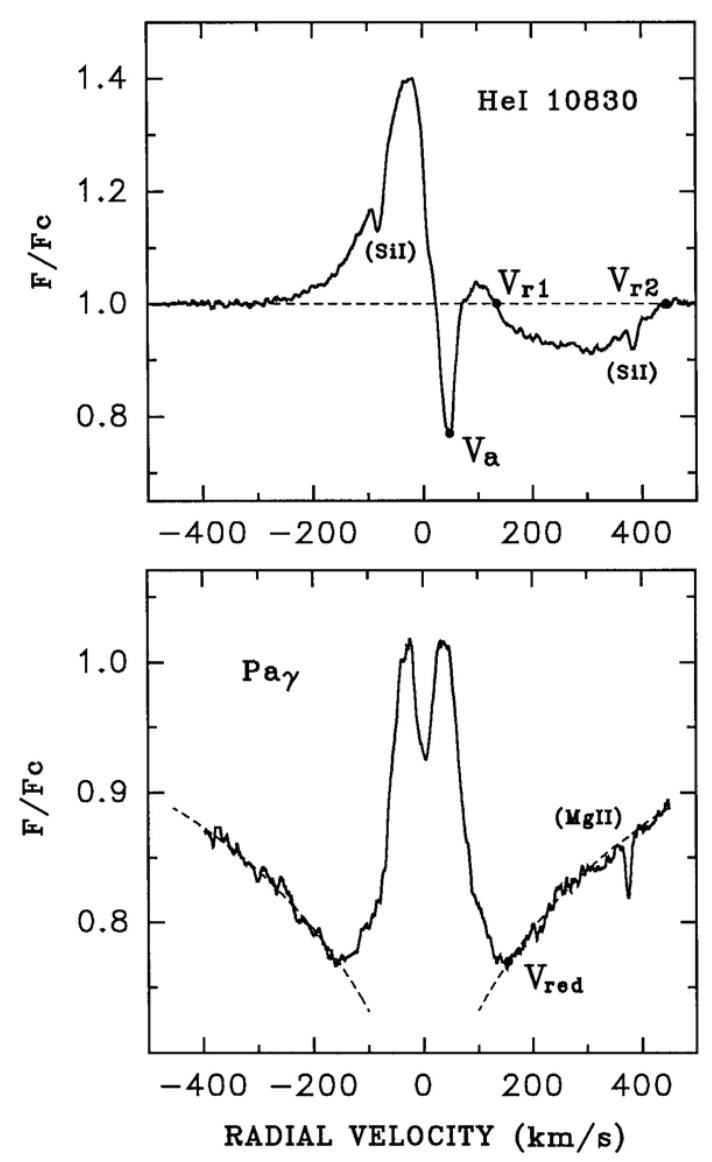

Figure 2. Typical profiles of the NIR CS lines He I $\lambda 10830$ and Pa $\gamma$ in the spectrum of HD 101412 shown in [18]. Parameters of the profiles used in the quantitative analysis are indicated. Credit: Schöller et al., A\&A, 592, A50, 2016, reproduced with permission (C) ESO.

The behavior of the lines confirms the described above structure of magnetospheric accretion in HD101412. The central absorption of the profiles of both lines originates from the disk itself. The infalling streams where the broad redshifted absorption on the He I $\lambda 10830$ line profile is forming screen the stellar disk near the regions of magnetic poles. The high-temperature region of the He I $\lambda 10830$ formation is geometrically thinner in comparison to Pa $\gamma$. The region of the Pa $\gamma$ line formation is much more extended, and the $\mathrm{Pa} \gamma$ line profile includes emission not only from the accreted flow, but also from the inner disk outside the magnetosphere. Due to this, the redshifted absorption, which is clearly seen in the He I $\lambda 10830$ line profile, is completely overlapped by a double-peaked emission from the inner disk in the case of the Pa $\gamma$ line profile.

Expecting that the variability of the He I $\lambda 10830$ and Pa $\gamma$ line parameters could be of cyclic character, as a result of profile modulation by the rotation of the star with its magnetosphere, to detect this variability we have chosen the following line parameters: the equivalent width $(\mathrm{EW})$ of both lines, the velocities of the blue $\left(V_{\mathrm{r} 1}\right)$ and red $\left(V_{\mathrm{r} 2}\right)$ edges of the redshifted absorption component of the He I $\lambda 10830$ line, and of the red boundary of the Pa $\gamma$ emission profile (see Figure 2).

The periodogram analysis of these five parameters was carried out using the method of fitting phase dependencies of the observed data for each value of the trial period $P$ with a sine for a range of $P$ from 5 to 80 days. Parameters of the sinusoids were determined by the least-square method. The periodograms constructed for three parameters of the He I $\lambda 10830$ line are shown in the left of Figure 3. We also calculated the noise periodogram 
in order to estimate the significance level of the separate peaks and to determine the window function (the details of the method can be found in $[19,20])$. The parameter $A / \sigma$ denotes the amplitude of the sinusoid in the unites of standard deviation of the residuals of the sine function fit for a given $P$.
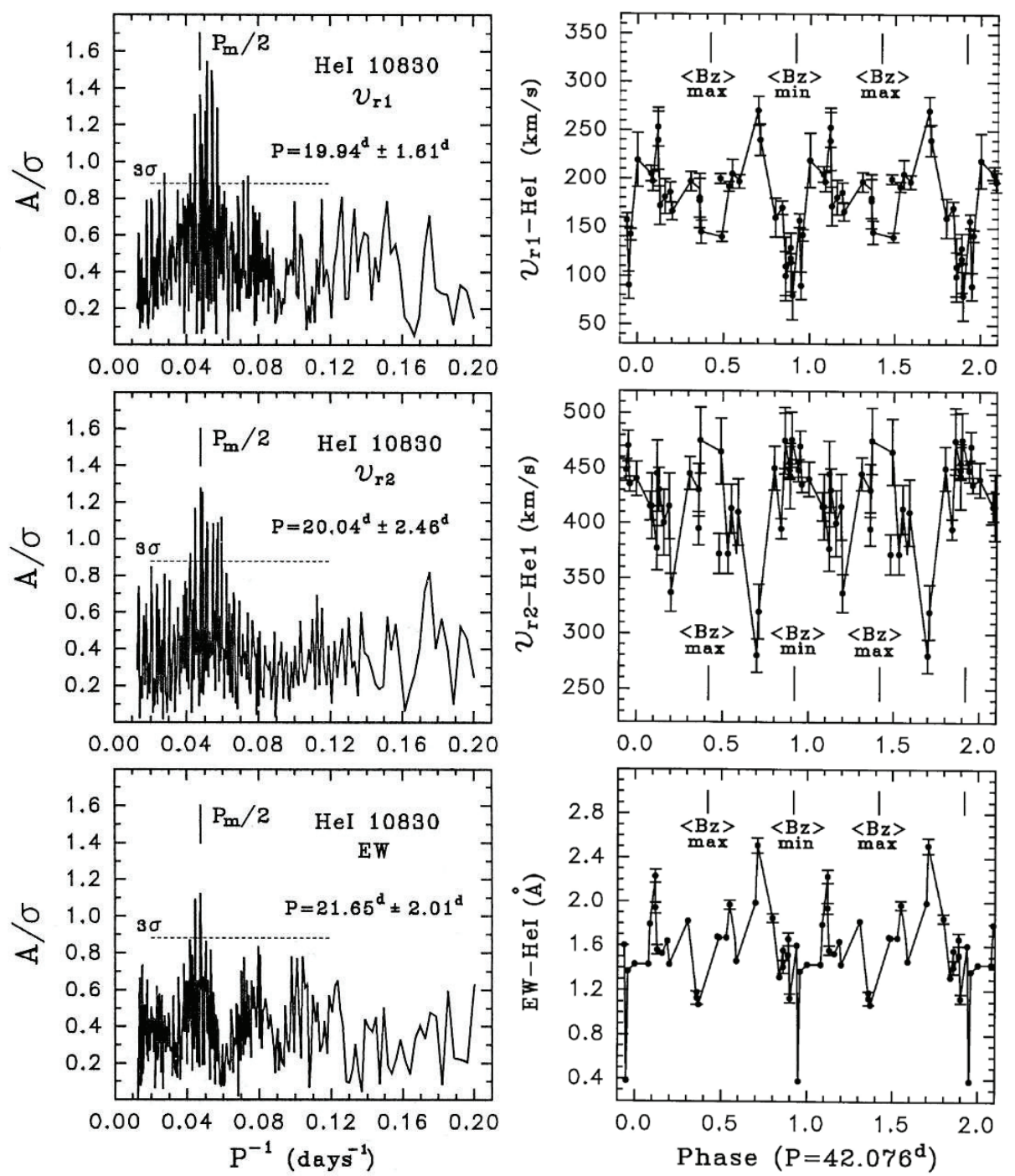

Figure 3. Left: $A / \sigma$ periodograms for different parameters of the He I $\lambda 10830$ line shown in Figure 2. The $3 \sigma$ significance levels are marked by dashed lines. Short vertical lines indicate the value corresponding to half of the rotation period $\left(P_{\text {rot }} / 2=21.04\right)$. Right: Phase dependencies of different line parameters over the rotation period $P_{\mathrm{m}}=42 \mathrm{~d}$ 076. The initial phase $\phi=0$ corresponds to MJD 52797.4. Credit: Schöller et al., A\&A, 592, A50, 2016, reproduced with permission $\odot$ ESO.

The reappearance of the same period in all periodograms can be considered as a criterion of its validity. All periodograms contain the peak near $P_{\mathrm{m}} / 2$ at a rather high significance level. The discrete structure of these wide peaks which is caused by a relatively small number of observations makes it possible to determine the mean value $P$ and the standard deviation of each peak (also presented on the left panels of Figure 3. The resulting period $P=20.53 \pm 1.68$ is close to half of the magnetic rotation period $P_{\mathrm{m}} / 2=21.038$.

The right of Figure 3 illustrates the phase dependencies of the He I $\lambda 10830$ line taken from $[17,18]$. As the MA model predicts, the $V_{r 2}$ parameter is at its maximum when $\left\langle B_{z}\right\rangle$ reaches its minimal or maximal value. In contrast to $V_{r 2}$, the parameters $V_{r 1}$ and EW demonstrate minimum values at these phases (when each of two accretion flows screens the star). 
We can conclude that our test delivers a positive result and that the behavior of the spectroscopic parameters for the lines originating close to the region of the disk/star interaction can be used for diagnostics of the magnetospheric character of the accretion from the disk onto the star.

\section{Signatures of the Magnetosphere around the Early Type Herbig B6e Star HD 259431}

HD 259431 is a Herbig B6e star associated with the reflection nebula NGC 2247. Its fundamental parameters are: $M=6.6 M_{\odot}, R=6.63 R_{\odot}, v \sin i=90 \mathrm{~km} / \mathrm{s}$ [21] and the accretion rate $\dot{M}=7.8 \times 10^{-7} M_{\odot} /$ yr [16]. No reliable magnetic field has been measured in this object $[11,22]$.

Our spectroscopic observations of the object were carried out at four observatories: (a) the Crimean AO (2.6 m Shajn telescope, echelle spectrograph); (b) ESO (2.2 m telescope, FEROS spectrograph); (c) the OAN SPM observatory in Mexico (2.1 m telescope, echelle spectrograph); and (d) the Kourovka UFU AO (1.2 m telescope, echelle spectrograph). More than 250 high-resolution spectra were obtained from 2010 to 2019.

HD 259431 demonstrates a remarkable type of spectral line variability. During three days, the double-peaked emission line $\mathrm{H} \gamma$ with a depression in the red wing transformed into a line with a PCyg-type profile (top of Figure 4). The same picture is observed in the Balmer lines from $\mathrm{H} \beta$ up to $\mathrm{H} \epsilon$. At epochs where we see a depression in the red wings of the emission Balmer lines, a broad absorption wing of He I $\lambda 5876$ is observed up to $+400 \mathrm{~km} / \mathrm{s}$ (see Figure 4 ). Such profile variability can be expected in the case of magnetospheric disk accretion, if the magnetic axis is inclined to the rotation axis. In this situation, the line of sight intersects repeatedly the region of the accretion flow or the wind zone, located at different magnetic latitudes. The existence of a magnetosphere is also evident from the strong increase of the red absorption wing of the He I $\lambda 5876$ line, as can be seen in Figure 4.

This picture could be validated if a search for periodic variations of the line parameters revealed a period close to the expected period of stellar rotation, $P_{\text {rot }}$. In our periodogram analysis, we used two spectroscopic parameters: $V_{\mathrm{bis}}(\mathrm{H} \beta)$ - a bisector velocity of the emission $\mathrm{H} \beta$ line profile at the continuum level $F_{\mathrm{c}}$-and $V_{\text {red }}(\mathrm{He} \mathrm{I})$-a velocity of the red boundary of the absorption wing of the He I $\lambda 5876$ line. Figure 5 illustrates that both parameters are in strong anti-correlation, with the coefficient $r=-0.805 \pm 0.049$. We used the standard Lomb-Scargle method [23], and tried to detect periodicity in the range of 2-3 days, which is likely to contain the $P_{\text {rot }}$ of the star, due to the previous spectrointerferometric estimation of $i$ in the range from $40^{\circ}$ to $60^{\circ}$ and $R=6.6 R \odot$ [21]. The significance level was determined by the method described in [24]. As one can see in the periodograms displayed in Figure 6, both parameters demonstrate a period $P=2.839 \mathrm{~d}$. The phase dependencies constructed for these two line parameters are presented in Figure 7. The dependencies look rather "noisy", with a large amplitude of scatter. This can be explained by the presence of other types of spectral variability that are not connected with stellar rotation, on the same timescales. As can be seen in Figure 7, the cyclic variations of both parameters take place in opposite phases. Adopting our estimation of $P=2.839 \mathrm{~d}$ as the most probable value of the rotation period, we can estimate the inclination angle $i$ as $48^{\circ} \pm 7^{\circ}$. 


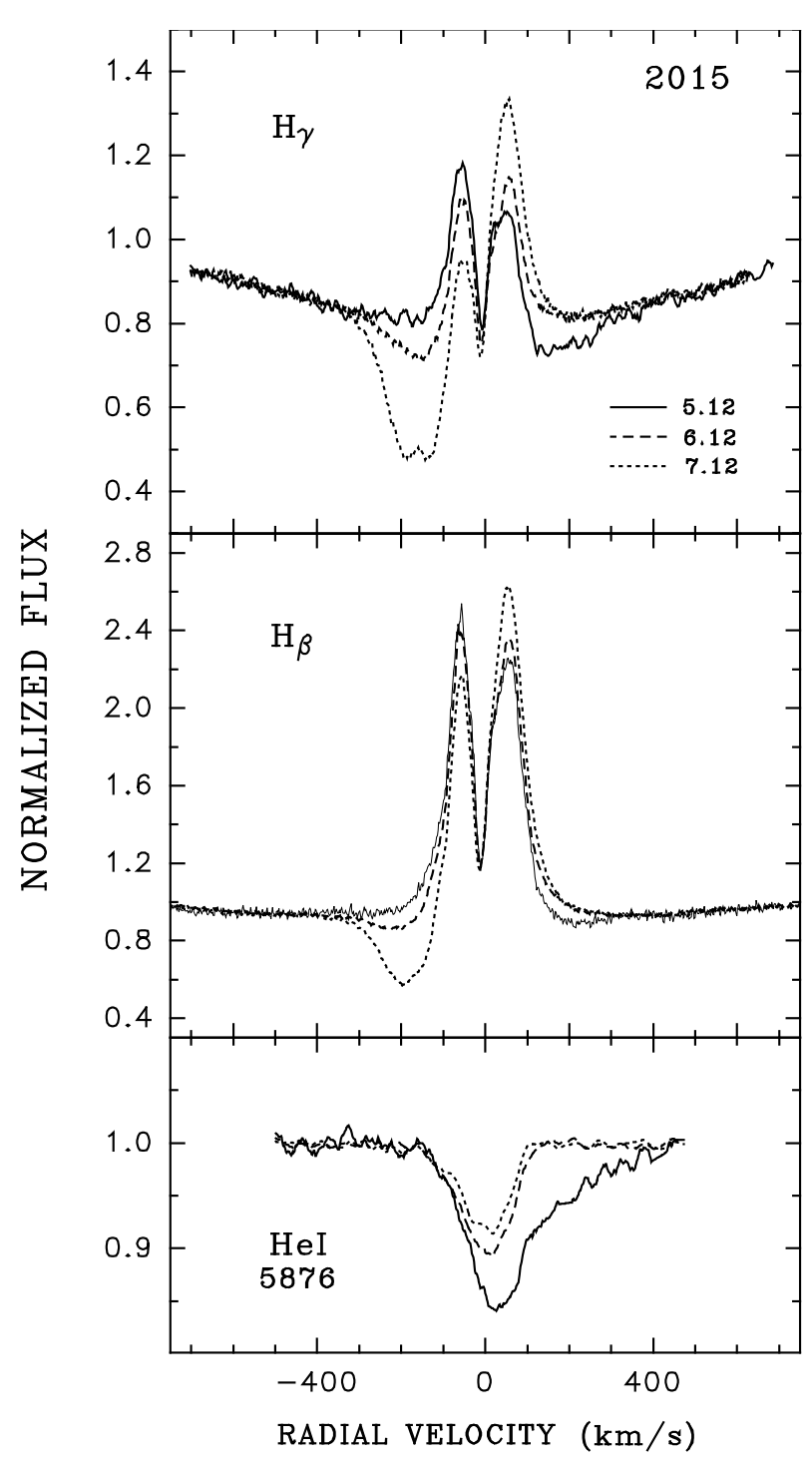

Figure 4. Variations of the line profiles for $\mathrm{H} \gamma, \mathrm{H} \beta$, and $\mathrm{He} \mathrm{I} \lambda 5876$ in the spectrum of HD 259431 during three epochs on 5-7 December 2015. X-scale is the same for all three plots.

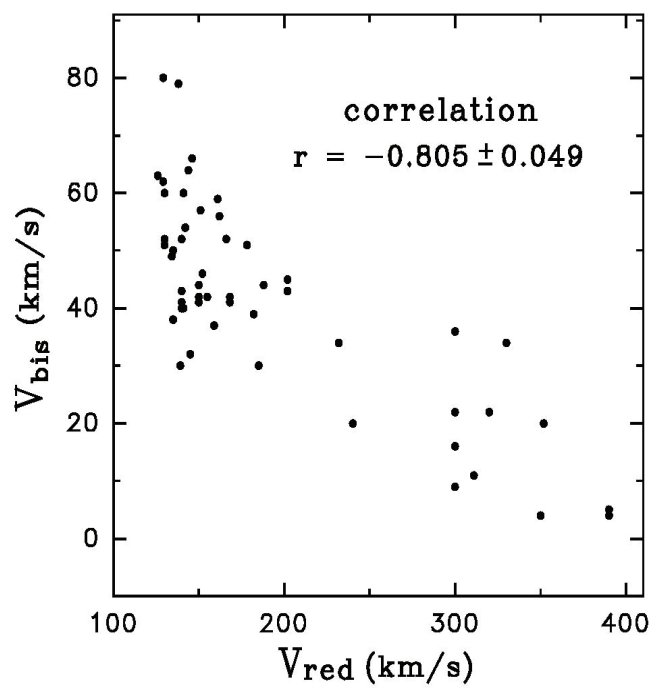

Figure 5. Dependence between the parameters $V_{\mathrm{bis}}(\mathrm{H} \beta)$ and $V_{\text {red }}(\mathrm{He} \mathrm{I} \lambda 5876)$ in the spectra of HD 259431. $r$ is the correlation coefficient. 


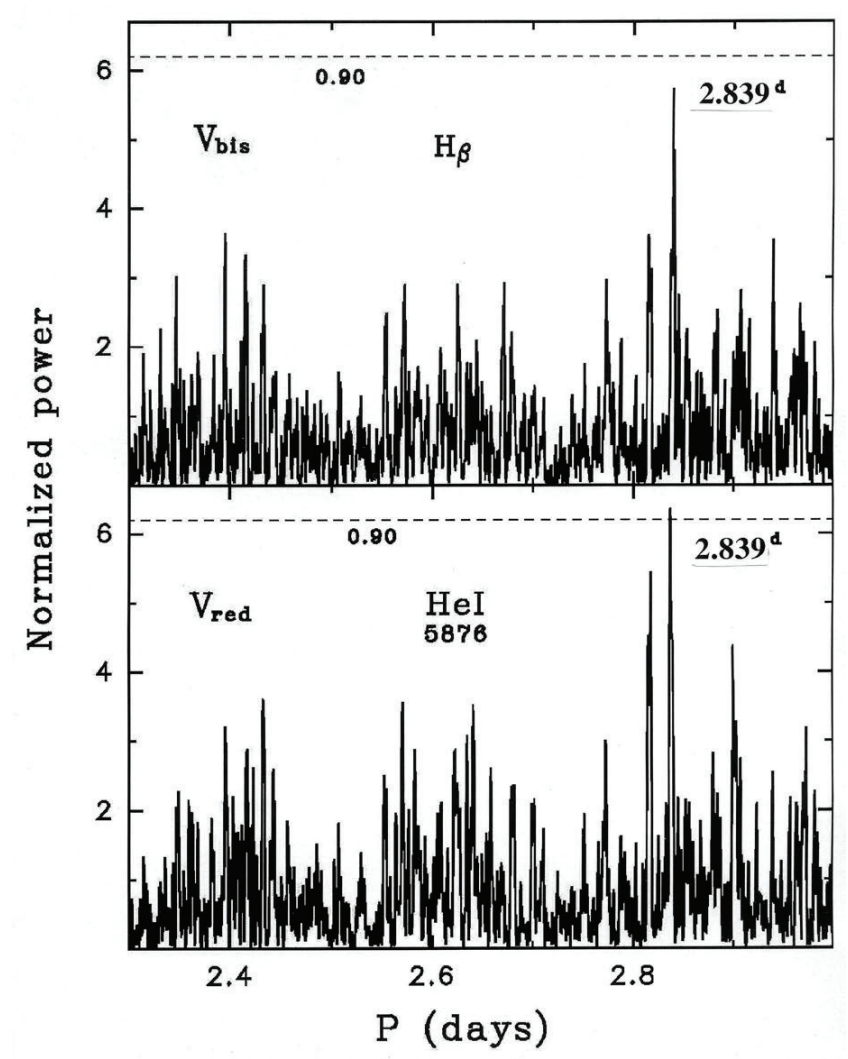

Figure 6. Lomb-Scargle periodogram for the parameters $V_{\text {bis }}(\mathrm{H} \beta)$ and $V_{\text {red }}(\mathrm{He}$ I $\lambda 5876)$ from the HD 259431 spectra. The same period, $P=2.839 \mathrm{~d}$, is visible in both periodograms.

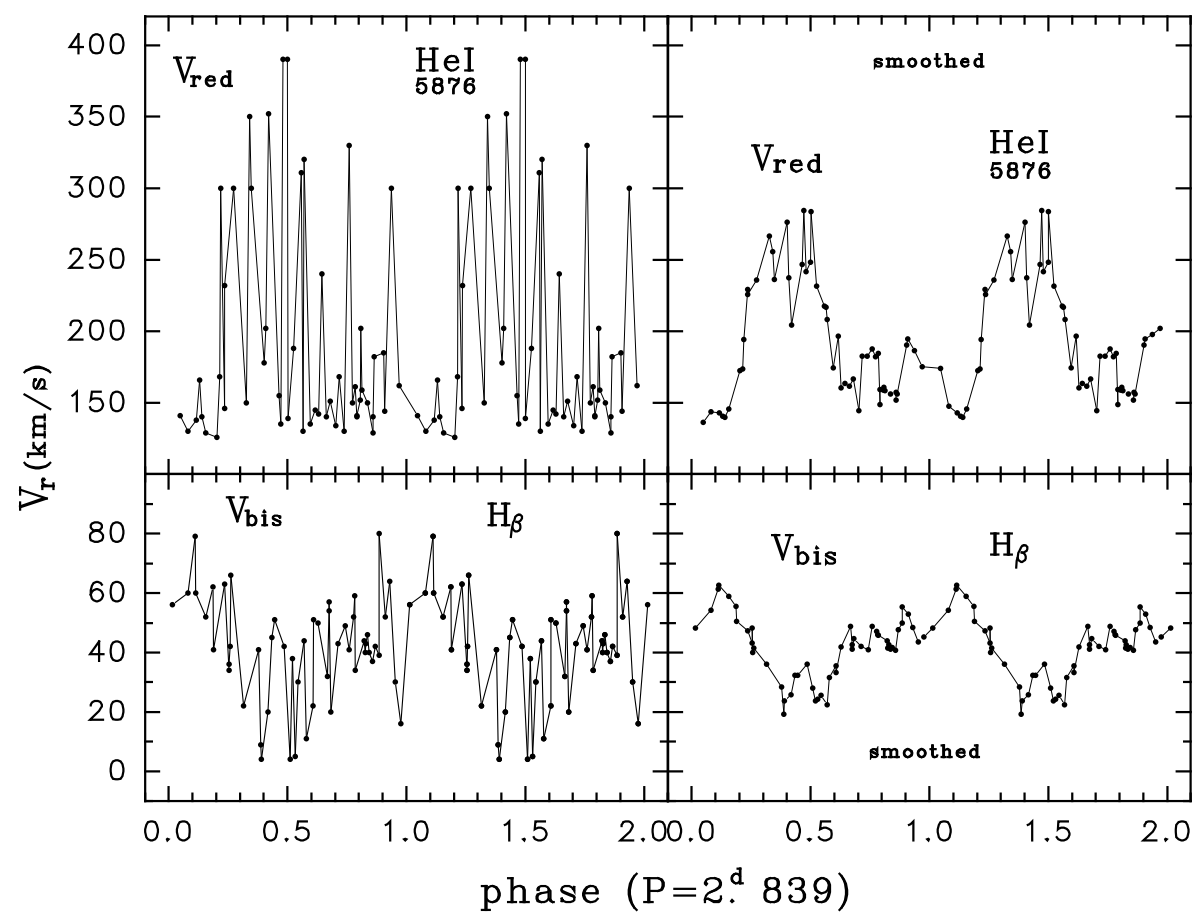

Figure 7. Phase dependencies of the parameters $V_{\mathrm{bis}}(\mathrm{H} \beta)$ and $V_{\text {red }}(\mathrm{He} \mathrm{I} \lambda 5876)$ from the HD 259431 spectra, constructed for the period $P=2.839 \mathrm{~d}$ (left) and smoothed over five data points (right).

Using the time when the parameter $V_{\text {red }}(\mathrm{He}$ I $\lambda 5876)$ reaches its maximum as the initial phase $\phi=0$, we can construct the phase variations of the profiles of some other CS lines in the spectrum of HD 259431. Figure 8 illustrates such variability for the lines $\mathrm{H} \delta$, He I $\lambda 6678$ 
and the OI $\lambda 7773$ triplet. All of them show the same behavior with the rotation phase as the lines $\mathrm{H} \beta$ and $\mathrm{He}$ I $\lambda 6678$. In general, the results of our spectroscopic investigation of HD 259431 confirm the presence of a magnetosphere. The estimated Alfvén radius of the object using Equation (1) is $R_{\mathrm{A}}=1.9 R$ (for $B=300 \mathrm{G}$ ), and $R_{\mathrm{A}}=1.5 R$ (for $B=200 \mathrm{G}$ ), which appear to be quite reasonable. The schematic picture of the disk/star interaction near the stellar surface is shown in Figure 9.

We would like to note that our result is the first detection of magnetospheric accretion among earlier-type Herbig Be stars (here: B6). This contradicts the conclusion of Cauley and Johns-Krull [25] that Herbig Be stars have no magnetospheres. For more details see Pogodin et al. [26].
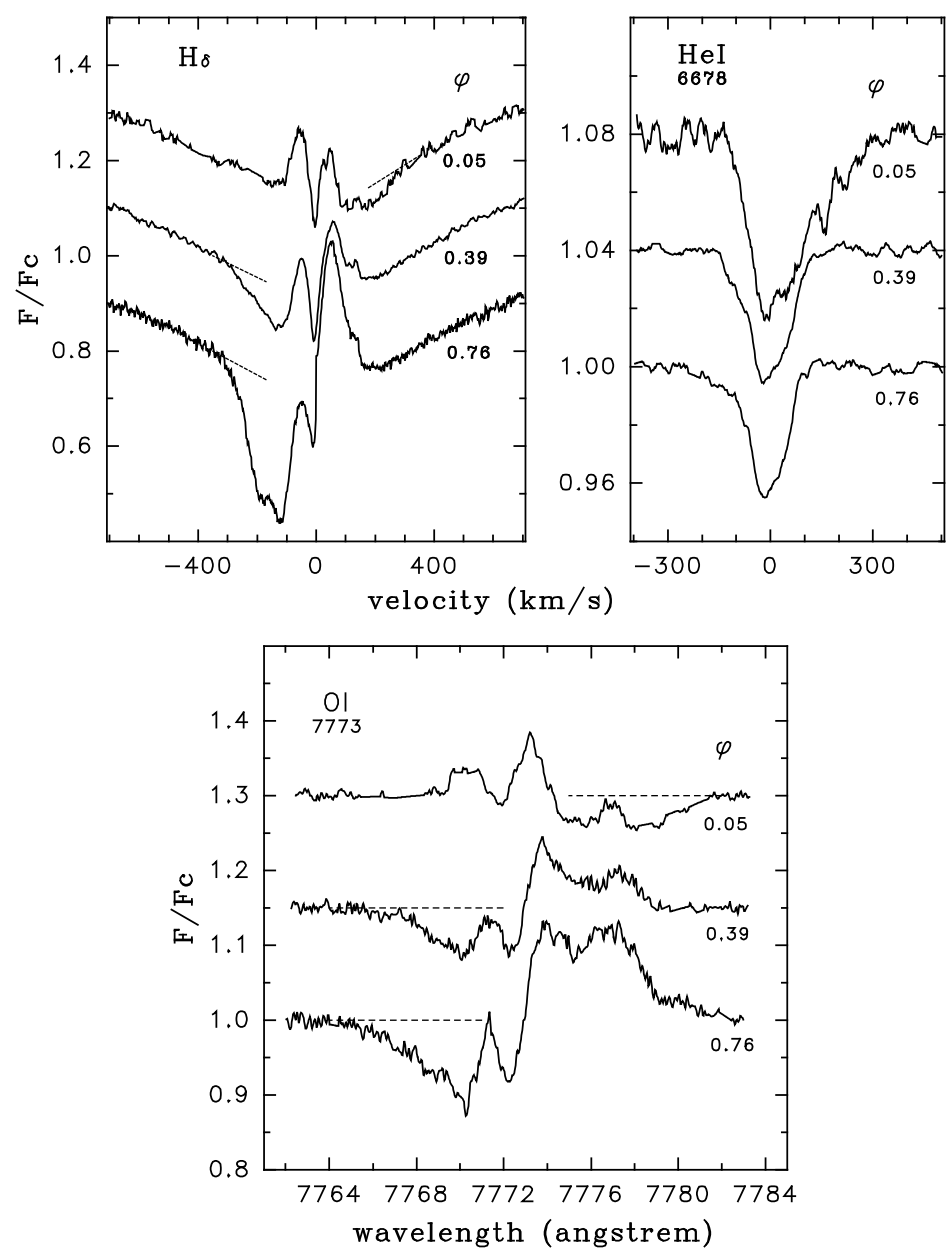

Figure 8. Profiles of the lines $\mathrm{H} \delta$, He I $\lambda 6678$ and OI $\lambda 7773$ observed in the spectra of HD 259431 at the phases of $0.05,0.39$ and 0.76 of the period $P=2.839 \mathrm{~d}$. The initial phase $\varphi=0$ corresponds to the epoch when the parameter $V_{\text {red }}(\mathrm{He}$ I $\lambda 5876)$ was maximal. 


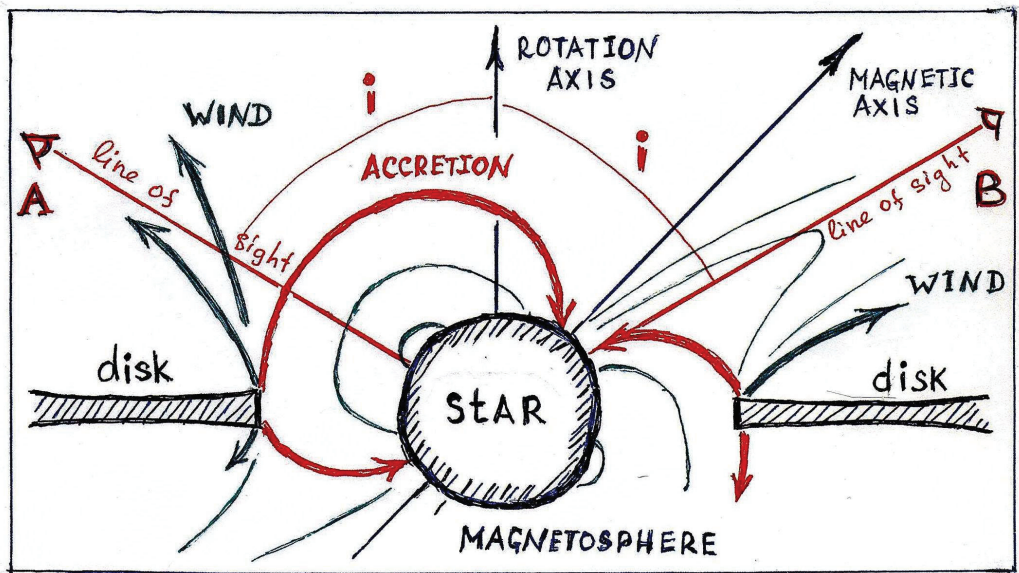

Figure 9. Schematic picture of the region of disk/star interaction near HD 259431, where the magnetic axis is inclined relative to the rotation axis. The star, disk, magnetosphere and wind zone are shown in the figure. The picture is presented in the reference frame of the star. Two directions of the line-of-sight are indicated. From direction A the line of sight intersects the wind zone at low magnetic latitudes, from direction $B$ the line of sight intersects the accretion stream inside the magnetosphere at high magnetic latitudes.

\section{The Young Magnetized Binary System HD 104237}

HD 104237 is a young binary system containing a Herbig Ae primary $\left(M_{1}=2.2 M_{\odot}\right)$ and a T Tauri secondary of $\mathrm{K} 2$ spectral type $\left(M_{2}=1.4 M_{\odot}\right)$. Böhm et al. [27] derived the orbital elements and velocity curves for the components. They estimated $P_{\text {orb }}=19.859 \mathrm{~d}$, $i_{\text {orb }}=17^{\circ}, e=0.665$, and $\gamma=13.9 \mathrm{~km} / \mathrm{s}$, constructing the radial velocity curves for both components. The basic stellar parameters of the system components were determined in a number of studies [27-30]. The most recent analysis by Cowley, Castelli and Hubrig [30] indicated $T_{\text {eff }}=8250 \mathrm{~K}, \log g=4.2, v \sin i=8 \mathrm{~km} / \mathrm{s}$ for the primary and $T_{\text {eff }}=4800 \mathrm{~K}$, $\log g=3.7$ and $v \sin i=12 \mathrm{~km} / \mathrm{s}$ for the secondary.

Such low projected velocities are beneficial for the detection of the magnetic field by the spectropolarimetric method. A possible presence of a weak magnetic field in HD 104237 (of the order of $50 \mathrm{G}$ ) was announced over 20 years ago by Donati at al. [31]. Later Wade et al. [22] did not confirm this detection. Hubrig et al. [7] estimated $\left\langle B_{\mathrm{Z}}\right\rangle=63 \pm 15 \mathrm{G}$ for the primary from a high-resolution HARPSpol spectrum, and, more recently, Järvinen et al. [32] reported a definite detection of a magnetic field $\left\langle B_{Z}\right\rangle=129 \pm 12 \mathrm{G}$ for the secondary T Tauri star and a marginal detection of $\left\langle B_{z}\right\rangle=13 \mathrm{G}$ for the primary.

The most recent HARPSpol spectropolarimetric analysis using the LSD and SVD methods allowed to measure the magnetic fields of the system's components more precisely. Eighty-eight spectra obtained in March 2015 were analyzed. The $\left\langle B_{z}\right\rangle$ value of the primary was found to be varying on different dates from $47 \pm 6 \mathrm{G}$ to $72 \pm 6 \mathrm{G}$ and that of the secondary from $609 \pm 27 \mathrm{G}$ to $124 \pm 13 \mathrm{G}$ [32].

The components of this binary system have a CS matter distribution in the region of the disk/star interaction that is different from expected for a single Herbig $\mathrm{Ae} / \mathrm{Be}$ star with a magnetosphere. Dunhill et al. [33] constructed a spatial structure of the CS material distribution using their smoothed particle hydrodynamics (SPH) simulations of the circumbinary disk around the system HD104237. The binary clears out a large cavity in the disk, which is not centered on the binary center of mass. Two streams of matter are accreting from the circumbinary disk onto the system components. The size of the two individual disks around the components is expected to be rather small due to tidal interaction. A connection of these accretion streams with the magnetic fields of both components is not clear, but in any case, local spots can be expected on the stellar surface in the stream impact regions. The rotation of such spots can modulate the photometric parameters of the object as well as the observed parameters of atmospheric lines, if the spectral data were obtained with sufficient accuracy. As a result, the method of diagnostics 
of local features on the stellar surface of the system components is the same as suggested for single objects that were tested for the presence of a magnetosphere.

The equivalent widths of the Stokes I LSD profiles of the metal lines in the spectrum of the primary component were used to search for periodicity corresponding to the expected rotation period $P_{\text {rot. }}$. The search was carried out using a non-linear least-square fit to multiple harmonics using the Levenberg-Marquardt method [34]. The periodogram is presented in Figure 10 (top). We also made a statistical test to check the null hypothesis on the absence of periodicity, i.e., to check the statistical significance of the fit [35]. The window function is also shown in the figure. The contribution of the secondary to the EWs at the phases of conjunction was removed from the measured values. As a result, we obtained a best period solution at $P=4.33717 \pm 0.00316 \mathrm{~d}$. Figure 10 (bottom) illustrates the phase dependence of the EWs constructed for the derived period $P=4.33717 \mathrm{~d}$. The nature of this periodicity is likely to be connected with the existence of temperature spots on the stellar surface.
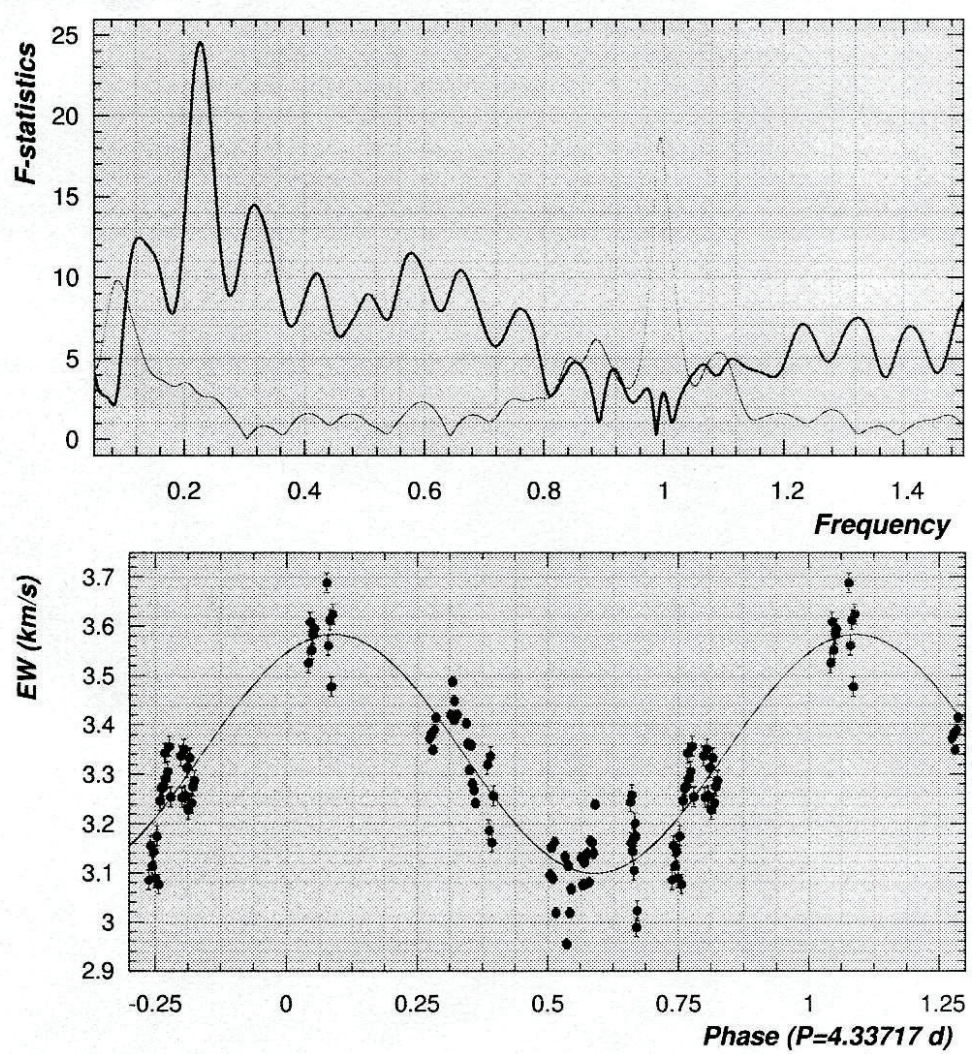

Figure 10. Top: Periodigram for the equivalent width measurements of the primary in HD 104237. The peak at $0.23057 \mathrm{~d}^{-1}$ corresponds to a period of $4.33717 \mathrm{~d}$. The window function is denoted by a dotted line. Bottom: EWs measured in the primary phased with the period of $4.33717 \mathrm{~d}$.

This estimate of the rotation period of the primary is in good agreement with the previous result by Böhm et al. [36], $P_{\text {rot }}=100 \pm 5 \mathrm{~h}$, which was derived from the analysis of CS H $\alpha$ line variations. This favors the idea that these spots on the stellar surface can be connected with inhomogeneities rotating in the CS media around the star, in other words, with the accretion flows inside the magnetosphere.

\section{The Herbig Ae/Be star HD 37806}

Our study of the Herbig Ae/Be star HD 37806 was also devoted to a search for a magnetosphere, but in this case we applied a different method, which is not using the modulation of the observational parameters by a hypothetical rotating azimuthal inhomogeneity. 
HD 37806 is an isolated Herbig star of A2-B8 type, associated with the Orion OB1b subgroup in the OB1 association. It has a $v \sin i=120 \mathrm{~km} / \mathrm{s}$ and no magnetic field has been detected so far [11,22]. Our spectroscopic observations of the object were carried out in November 2012 with the Coudé spectrograph ASP-14 installed at the $2.6 \mathrm{~m}$ Shajn telescope of the Crimean AO. Twenty spectra near the He I $\lambda 5876$ line were obtained during the two nights of $8 / 9$ and $11 / 12$ November. The analysis of the variability observed during each night has shown that the profile of this line originating in the high-temperature zone of the disk/star interaction demonstrates intensity variations in the form of standing waves in the region of the red absorption (see Figure 11, for the second observing night). These results are presented in more detail in Pogodin et al. [37].

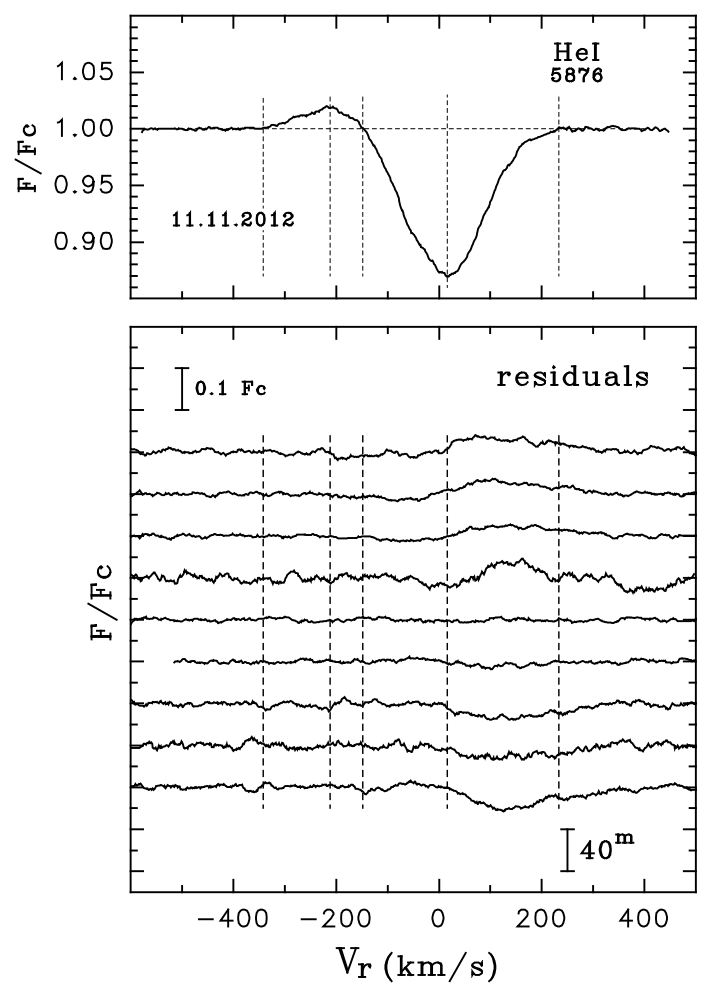

Figure 11. Top: Normalized profiles of the He I $\lambda 5876$ line observed in the spectrum of HD 37806 on 11 November 2012. Bottom: Residual profiles constructed relative to the nightly mean. Both intensity and time scale are indicated. The time increases from top to bottom.

Extensive model calculations have shown that such variability can appear only if a rotating local stream intersects the line-of-sight, which is orthogonal to the Surfaces of Equal Radial Velocities (SERVs) of the moving gas. In the case of a purely Keplerian accretion disk (see left of Figure 12) or a disk with radial motion (see right of Figure 12), the SERVs are not orthogonal to the line-of-sight in the region between the star and the observer. In this case the short-term variations would appear as running waves on the residual plots. Figures 12 and 13 were first presented in slightly different form in the paper by Pogodin et al. [37]. 

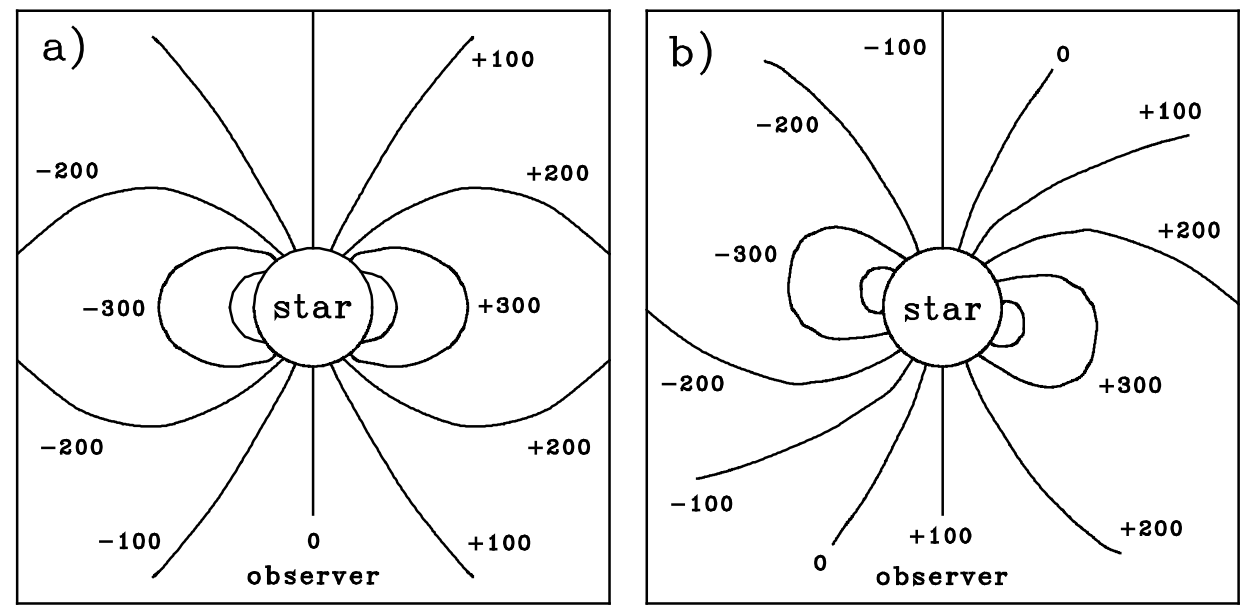

Figure 12. Picture of the SERVs distribution in the disk plane near the star. The case (a) is for the Keplerian disk; the case (b) is the same as (a) but with a radial velocity of the accretion onto the star of $V=100 \mathrm{~km} / \mathrm{s}$. The corresponding values of the radial velocities are indicated near each SERV in the framework of the observer.

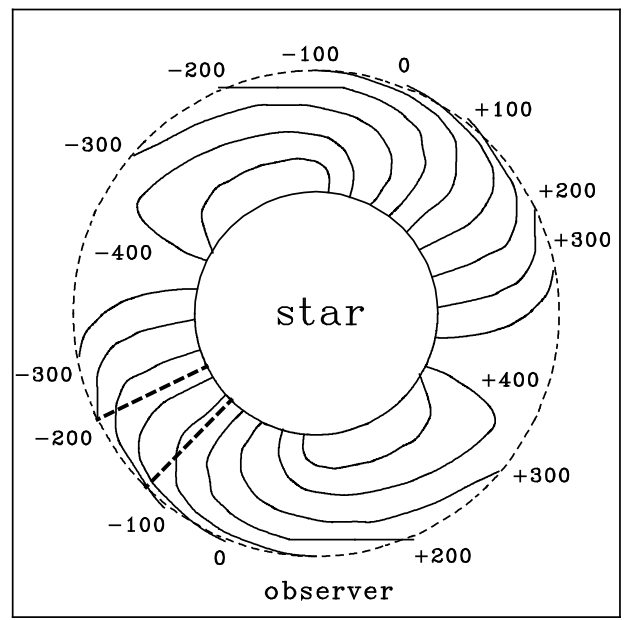

Figure 13. The same as Figure 12, but for the MA model. The parameters of the model are indicated in Section 6.

Only in the case of the MA scenario the SERVs intersecting the line-of-sight can become orthogonal to it. Figure 13 is constructed for the SERVs picture of the simplest geometric model $\left(i=90^{\circ}, \beta=90^{\circ}\right)$ such as in the case of HD 101412 (see Section 3). The figure illustrates the SERVs' orientation corresponding to this model. Inside the magnetosphere, we assume the rigid rotation velocity law $V_{\text {rot }}(r)=U_{0}(r / R)$, and the radial velocity $V_{\mathrm{r}}=V_{0}\left(R / r-R / R_{\mathrm{A}}\right)$ for the freefall regime, where $U_{0}=160 \mathrm{~km} / \mathrm{s}$ is the equatorial rotation velocity on the stellar surface, and $V_{0}$, the radial velocity at the outer magnetospheric boundary, where $R_{\mathrm{A}}=2 R$, is equal to $635 \mathrm{~km} / \mathrm{s}$, the terminal velocity for the object. Furthermore, we calculated the He I $\lambda 5876$ residuals for this model at different rotation phases, adopting the gas to be optically thick in this spectral line and the source function to be equal to 0.1 of the stellar intensity (see Figure 14).

In the general case, for any geometry and orientation of the magnetic field configuration the character of the SERVs is expected to be similar. It is determined by the specific kinematics of the gas inside of the magnetosphere: the radial velocity is minimal near the outer boundary of the magnetosphere and maximal near the stellar surface (with free-fall motion). Its rotation velocity is, on the contrary, maximal at the outer boundary of the magnetosphere and minimal near the stellar surface (rigidly rotating with the star). 

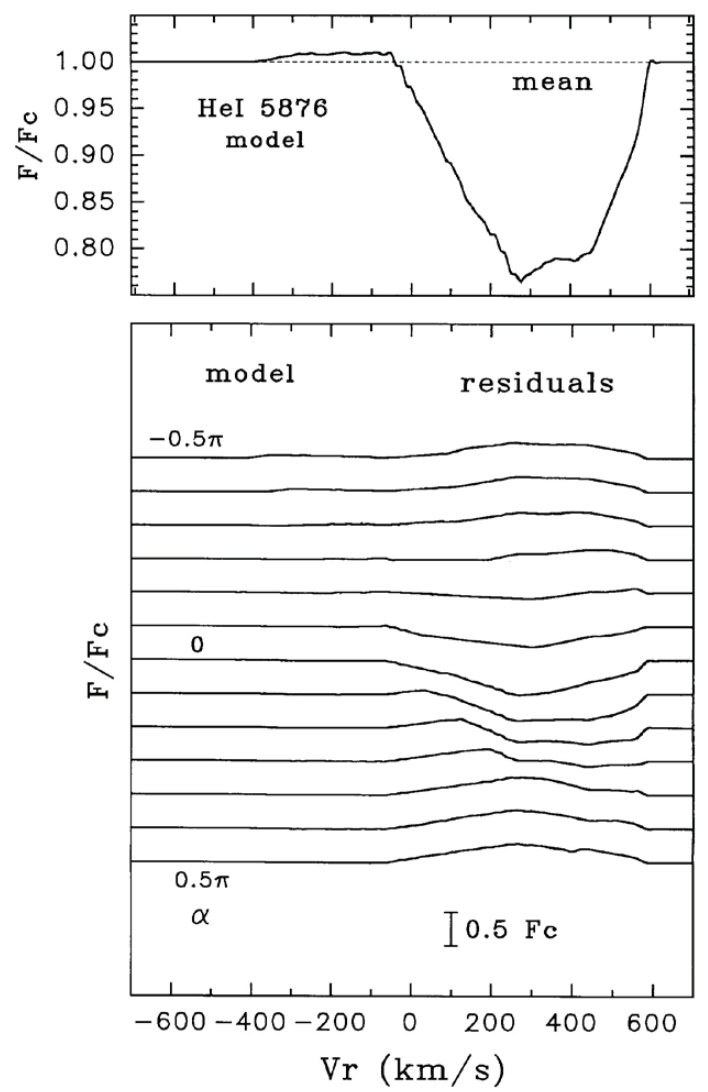

Figure 14. The same as Figure 11, but for the model described in detail in Section 6. $\alpha$ is an angle between the axis of the rotating stream and the line of sight.

We conclude that this type of short-term spectroscopic variability can be considered as an additional signature of the MA scenario. Besides that, the estimation of $R_{\mathrm{A}}$ using Equation (1) shows reasonable values: $R_{\mathrm{A}}=1.7-2.2 R$ for $B=200-300 \mathrm{G}$, respectively. In these calculations, we used the following values: $M=4 M_{\odot}, R=4.6 R_{\odot}$, and a mass accretion rate $\dot{M}=1.4 \times 10^{-7} M_{\odot} /$ yr.

\section{Conclusions}

We consider several diagnostical methods for the magnetospheric accretion in Herbig $\mathrm{Ae} / \mathrm{Be}$ stars, which are surrounded by accretion disks. The detection of a magnetosphere can be considered as a supplement to the widely used spectropolarimetric observations to measure stellar magnetic fields.

The magnetosphere is a CS region near the star where the disk accretion onto the star is completely governed by the magnetic field of the star. If the magnetic axis is inclined relative to the rotation axis, a global azimuthal inhomogeneity is likely to be formed. Its rotation can modulate different spectral or photometric parameters of the object under investigation. The registration of such a modulation in a form of cyclic parameter variations is the most common way to reveal the presence of the MA scenario of disk/star interaction. Besides that, the moving gas inside the magnetosphere has a specific kinematics, the radial velocity corresponds to the free-fall regime, and the gaseous streams rotate rigidly with the star.

These features of the magnetospheric accretion have been used in our work. Four cases of MA diagnostics have been considered in this paper. These are: (a) HD 101412-a slowly rotating Herbig Ae star with an anomalously strong magnetic field; (b) the early type Herbig B6e star HD 259431; (c) the young binary system HD 104237 consisting of a Herbig Ae star and a T Tauri K2-type star; and (d) the Herbig Ae/Be star HD37806, where signs of the MA disk/star interaction were found from a particular type of short-term variability of the He I $\lambda 5876$ line profile during one night rather than from a search for 
periodicities in the observed parameter variations. The results of our investigation was positive in each of the four cases. We have found that even the two rapidly rotating objects HD 259431 and HD 37806 demonstrate signatures of magnetospheric accretion, i.e., they should have significant magnetic fields. The commonly recognized spectropolarimetric method was ineffective to detect magnetic fields in these two stars.

We hope that the application of MA diagnostics considered in this paper to other Herbig Ae/Be stars will allow to significantly increase the percentage of stars that possess magnetic fields among these objects.

Author Contributions: M.P.-conceptualization, observations at the $\mathrm{CrAO}$, data reduction and analysis and writing-original draft preparation; N.D.--reduction of the ESO data, synthetic spectra calculation and writing - editing; N.B.-observations at the OAN SPM and writing-LaTeX draft preparation, review and editing; S.H., M.S. and S.J.- observations at the ESO, acquisition of archive data, data reduction and analysis and writing-editing; S.P., O.K. and I.A.-observations at the $\mathrm{CrAO}$ and spectral data reduction. All authors have read and agreed to the published version of the manuscript.

Funding: M.A.P. and N.G.B. acknowledge the financial support of the Ministry of Science and Higher Education (grant no. 075-15-2020-780 “Exoplanets-4").

Data Availability Statement: The data underlying this article are available on request from the authors.

Acknowledgments: The authors are grateful to the reviewers for useful comments.

Conflicts of Interest: The authors declare no conflict of interest.

\section{References}

1. Herbig, G.H. The Spectra of Be- and Ae-Type Stars Associated with Nebulosity. Astrophys. J. Suppl. 1960, 4, 337. [CrossRef]

2. Finkenzeller, U.; Mundt, R. The Herbig Ae/Be stars associated with nebulosity. Astron. Astrophys. Suppl. 1984, 55, 109-141.

3. The, P.S.; de Winter, D.; Perez, M.R. A new catalogue of members and candidate members of the Herbig Ae/Be (HAEBE) stellar group. Astron. Astrophys. Suppl. 1994, 104, 315-339.

4. Gullbring, E.; Hartmann, L.; Briceño, C.; Calvet, N. Disk Accretion Rates for T Tauri Stars. Astrophys. J. 1998, 492, 323-341. [CrossRef]

5. Hubrig, S.; Schöller, M.; Yudin, R.V. Magnetic fields in Herbig Ae stars. Astron. Astrophys. 2004, 428, L1-L4. [CrossRef]

6. Hubrig, S.; Stelzer, B.; Schöller, M.; Grady, C.; Schütz, O.; Pogodin, M.A.; Curé, M.; Hamaguchi, K.; Yudin, R.V. Searching for a link between the magnetic nature and other observed properties of Herbig Ae/Be stars and stars with debris disks. Astron. Astrophys. 2009, 502, 283-301. [CrossRef]

7. Hubrig, S.; Ilyin, I.; Schöller, M.; Lo Curto, G. HARPS spectropolarimetry of Herbig Ae/Be stars. Astron. Nachrichten 2013, 334, 1093. [CrossRef]

8. Hubrig, S.; Carroll, T.A.; Scholler, M.; Ilyin, I. The prevalence of weak magnetic fields in Herbig Ae stars: The case of PDS 2. Mon. Not. RAS 2015, 449, L118-L122. [CrossRef]

9. Wade, G.A.; Drouin, D.; Bagnulo, S.; Landstreet, J.D.; Mason, E.; Silvester, J.; Alecian, E.; Böhm, T.; Bouret, J.C.; Catala, C.; et al. Discovery of the pre-main sequence progenitors of the magnetic Ap/Bp stars? Astron. Astrophys. 2005, 442, L31-L34. [CrossRef]

10. Alecian, E.; Catala, C.; Wade, G.A.; Donati, J.F.; Petit, P.; Landstreet, J.D.; Böhm, T.; Bouret, J.C.; Bagnulo, S.; Folsom, C.; et al. Characterization of the magnetic field of the Herbig Be star HD200775. Mon. Not. RAS 2008, 385, 391-403. [CrossRef]

11. Alecian, E.; Wade, G.A.; Catala, C.; Grunhut, J.H.; Landstreet, J.D.; Bagnulo, S.; Böhm, T.; Folsom, C.P.; Marsden, S.; Waite, I. A high-resolution spectropolarimetric survey of Herbig Ae/Be stars-I. Observations and measurements. Mon. Not. RAS 2013, 429, 1001-1026. [CrossRef]

12. Moss, D. The survival of fossil magnetic fields during pre-main sequence evolution. Astron. Astrophys. 2003, $403,693-697$. [CrossRef]

13. Hubrig, S.; Järvinen, S.P.; Schöller, M.; Carroll, T.A.; Ilyin, I.; Pogodin, M.A. Observations of Magnetic Fields in Herbig Ae/Be Stars. In Physics of Magnetic Stars, Proceedings of a Conference Held at Special Astrophysical Observatory, Nizhny Arkhyz, Russia, 1-5 October 2018; Kudryavtsev, D.O., Romanyuk, I.I., Yakunin, I.A., Eds., Astronomical Society of the Pasific: San Francisco, CA, USA, 2019; Volume 518, p. 18.

14. Camenzind, M. Magnetized Disk-Winds and the Origin of Bipolar Outflows. Rev. Mod. Astron. 1990, 3, 234-265. [CrossRef]

15. Wang, Y.M. Location of the Inner Radius of a Magnetically Threaded Accretion Disk. Astrophys. J. Lett. 1996, 465, L111-L113. [CrossRef]

16. Donehew, B.; Brittain, S. Measuring the Stellar Accretion Rates of Herbig Ae/Be Stars. Astron. J. 2011, 141, 46. [CrossRef] 
17. Hubrig, S.; Mikulá v sek, Z.; González, J.F.; Schöller, M.; Ilyin, I.; Curé, M.; Zejda, M.; Cowley, C.R.; Elkin, V.G.; Pogodin, M.A.; et al. Rotationally modulated variations and the mean longitudinal magnetic field of the Herbig Ae star HD 101412. Astron. Astrophys. 2011, 525, L4. [CrossRef]

18. Schöller, M.; Pogodin, M.A.; Cahuasquí, J.A.; Drake, N.A.; Hubrig, S.; Petr-Gotzens, M.G.; Savanov, I.S.; Wolff, B.; González, J.F.; Mysore, S.; et al. Spectroscopic signatures of magnetospheric accretion in Herbig Ae/Be stars. I. The case of HD 101412. Astron. Astrophys. 2016, 592, A50. [CrossRef]

19. Zechmeister, M.; Kürster, M. The generalised Lomb-Scargle periodogram. A new formalism for the floating-mean and Keplerian periodograms. Astron. Astrophys. 2009, 496, 577-584. [CrossRef]

20. Alvarado-Gómez, J.D.; Hussain, G.A.J.; Grunhut, J.; Fares, R.; Donati, J.F.; Alecian, E.; Kochukhov, O.; Oksala, M.; Morin, J.; Redfield, S.; et al. Activity and magnetic field structure of the Sun-like planet-hosting star HD 1237. Astron. Astrophys. 2015, 582, A38. [CrossRef]

21. Kraus, S.; Preibisch, T.; Ohnaka, K. Detection of an Inner Gaseous Component in a Herbig Be Star Accretion Disk: Near- and Mid-Infrared Spectrointerferometry and Radiative Transfer modeling of MWC 147. Astrophys. J. 2008, 676, 490-508. [CrossRef]

22. Wade, G.A.; Bagnulo, S.; Drouin, D.; Landstreet, J.D.; Monin, D. A search for strong, ordered magnetic fields in Herbig Ae/Be stars. Mon. Not. RAS 2007, 376, 1145-1161. [CrossRef]

23. Scargle, J.D. Studies in astronomical time series analysis. II. Statistical aspects of spectral analysis of unevenly spaced data. Astrophys. J. 1982, 263, 835-853. [CrossRef]

24. Horne, J.H.; Baliunas, S.L. A Prescription for Period Analysis of Unevenly Sampled Time Series. Astrophys. J. 1986, $302,757$. [CrossRef]

25. Cauley, P.W.; Johns-Krull, C.M. Diagnosing Mass Flows around Herbig Ae/Be Stars Using the He I $\lambda 10830$ Line. Astrophys. J. 2014, 797, 112. [CrossRef]

26. Pogodin, M.A.; Pavlovskiy, S.E.; Drake, N.A.; Beskrovnaya, N.G.; Borges Fernandes, M.; Pereira, S.B.; Kozlova, O.V.; Alekseev, I.Y.; Valyavin, G.G.; Miroshnichenko, A.S.; et al. The Spectroscopic Variability of the Herbig Be Star HD 259431. In Physics of Magnetic Stars, Proceedings of a Conference Held at Special Astrophysical Observatory, Nizhny Arkhyz, Russia, 1-5 October 2018; Kudryavtsev, D.O., Romanyuk, I.I., Yakunin, I.A., Eds., Astronomical Society of the Pasific: San Francisco, CA, USA, 2019; Volume 518, p. 18.

27. Böhm, T.; Catala, C.; Balona, L.; Carter, B. Spectroscopic monitoring of the Herbig Ae star HD 104237. I. Multiperiodic stellar oscillations. Astron. Astrophys. 2004, 427, 907-922. [CrossRef]

28. Grady, C.A.; Woodgate, B.; Torres, C.A.O.; Henning, T.; Apai, D.; Rodmann, J.; Wang, H.; Stecklum, B.; Linz, H.; Williger, G.M.; et al. The Environment of the Optically Brightest Herbig Ae Star, HD 104237. Astrophys. J. 2004, 608, 809-830. [CrossRef]

29. Fumel, A.; Böhm, T. Spectroscopic monitoring of the Herbig Ae star HD 104237. II. Non-radial pulsations, mode analysis, and fundamental stellar parameters. Astron. Astrophys. 2012, 540, A108. [CrossRef]

30. Cowley, C.R.; Castelli, F.; Hubrig, S. The Herbig Ae SB2 system HD 104237. Mon. Not. RAS 2013, 431, 3485-3493. [CrossRef]

31. Donati, J.F.; Semel, M.; Carter, B.D.; Rees, D.E.; Collier Cameron, A. Spectropolarimetric observations of active stars. Mon. Not. RAS 1997, 291, 658-682. [CrossRef]

32. Järvinen, S.P.; Carroll, T.A.; Hubrig, S.; Ilyin, I.; Schöller, M.; Drake, N.A.; Pogodin, M.A. The two magnetic components in the Herbig Ae SB2 system HD 104237. Mon. Not. RAS 2019, 486, 5499-5503. [CrossRef]

33. Dunhill, A.C.; Cuadra, J.; Dougados, C. Precession and accretion in circumbinary discs: The case of HD 104237. Mon. Not. RAS 2015, 448, 3545-3554. [CrossRef]

34. Press, W.H.; Teukolsky, S.A.; Vetterling, W.T.; Flannery, B.P. Numerical Recipes in FORTRAN. The Art of Scientific Computing; Cambridge University Press: Cambridge, UK, 1992.

35. Seber, G.A.F. Linear Regression Analysis; Wiley Press: New York, NY, USA, 1977.

36. Böhm, T.; Dupret., M.A.; Aynedjian, H. Seismology and rotation of the Herbig Ae star HD 104237 . Mem. Soc. Astron. Ital. 2006, $77,362$.

37. Pogodin, M.A.; Kozlova, O.V.; Alekseev, I.Y.; Pavlovskiy, S.E. Short-Term Spectral Variability of the Herbig Ae/Be Star HD37806. Astrophysics 2019, 62, 18-34. [CrossRef] 